\title{
Schriftverkehr auf der Datenautobahn: Besonderheiten der schriftlichen Kommunikation im Internet
}

\author{
Angelika Storrer ${ }^{1}$
}

\section{Fragestellung}

Ob das Internet die deutsche Sprache verändert, gehört zu den häufigsten Fragen, die Journalisten und sprachinteressierte Laien stellen, wenn man sich als internetkundige Linguistin zu erkennen gibt. Genaugenommen ist die Frage mit ,nein“ zu beantworten, denn natürlich können nicht Medien die Sprache verändern, sondern nur die Sprachteilnehmer, die diese Medien zur Kommunikation nutzen. Allerdings schaffen neue Medien meist auch neue Randbedingungen für die Rezeption und Produktion von Sprache, was sich auf Dauer auf die Gepflogenheiten der Sprachteilnehmer auswirkt und insofern - von einer abstrakten Außensicht her gesehen - tatsächlich zu Veränderungen sprachlicher Merkmale führt. Am besten ist dieser Zusammenhang für ein Medium untersucht, das - bei aller Internet-Euphorie - bislang die einschneidendsten Auswirkungen auf gesellschaftliche Entwicklungen hatte: Die Schrift. Die Einführung der Schrift erleichterte die Externalisierung und Verdauerung von Wissen erheblich und legte den Grundstein für das, was heute als Informations- und Wissensgesellschaft bezeichnet wird (vgl. Ehlich, 1994; Koch \& Oesterreicher, 1994).

Auch das Internet ist bislang noch ein schriftdominiertes Medium, d.h., die Mehrzahl der im Netz gespeicherten und übermittelten Daten ist schriftlich kodiert. Und da diese Daten auf einfache Weise archiviert und abgerufen werden können, wird auch schnell offenbar, was hinter der Frage zum Sprachwandel durch das Internet steckt: Was an schriftlichen Produkten über die Datenautobahn geschickt wird, weicht vielfach in auffälliger Weise von den Erwartungen ab, die man normalerweise schriftlichen Texten entgegenbringt. Die in Abbildung 3 und 4 gezeigten Bildschirmschnappschüsse illustrieren, warum in Publikationen zur Netzkommunikation von ,gekritzelten Telefonaten“, „elektronischen Kaffeekränzchen“, „virtuellen Gesprächen“, vom "Geschwätz im globalen Dorf" die Rede ist, warum die Produkte als „,verschriftete Mündlichkeit oder mündliche Schriftlichkeit“ (Günther \& Wyss, 1996, S. 82) bezeichnet werden.

1 storrer@ids-mannheim.de 
Das Internet verändert offensichtlich etwas im Umgang mit Schrift, verschiebt das Verhältnis von Sprech- und Schreibsprache. Worin besteht aber diese Verschiebung genau, handelt es sich dabei überhaupt um eine durch das neue Medium induzierte Entwicklung? Schließlich gilt es als generelle Tendenz der Sprachentwicklung im 20. Jahrhundert, daß sich gesprochene und geschriebene Sprache einander annähern (vgl. Sieber, 1998), daß eine „Epoche beispiellos starker Schriftlichkeit" (Polenz, 1999, S. 37) im Laufe des 20. Jahrhunderts durch eine ,teilweise Reoralisierung der öffentlichen Kommunikation" (Polenz, 1999, S. 39) abgelöst wird. Um diese Frage dreht sich der vorliegende Beitrag.

Zunächst werde ich in Abschnitt 2 eine terminologische Präzisierung der Ausdrücke „Mündlichkeit“ bzw. „Schriftlichkeit“ übernehmen, die sich in der modernen Schriftlichkeitsforschung als hilfreich und klärend erwiesen hat: Die Unterscheidung zwischen medialer Mündlichkeit und Schriftlichkeit einerseits und konzeptioneller Mündlichkeit und Schriftlichkeit andererseits. Auf dieser Basis lassen sich dann die Merkmale benennen, die als Indikatoren für eine „Vermündlichung“ von Schriftsprache an der Textoberfläche greifbar sind. In Abschnitt 3 werden dann drei Kommunikationsformen des Internet - Email, Foren (Newsgroups) und Chat - im Hinblick auf diese Sprachmerkmale untersucht, wobei der Schwerpunkt auf der Frage liegt, inwieweit sich die Merkmale der Mündlichkeit auf das jeweilige kommunikative Setting und die verfügbaren Softwarefunktionen zurückführen lassen. In Abschnitt 4 geht es dann um Tendenzen im Umgang mit Schriftlichkeit, die für alle Internet-Dienste und mithin für den kommunikativ-sozialen Stil (i.S. von Kallmeyer, 1995) der „Netzbürger“ charakteristisch sind. Auf dieser Basis wird abschließend die Frage wieder aufgegriffen und beantwortet, inwieweit die mündlichen Tendenzen in der schriftlichen Internet-Kommunikation eine qualitativ neue Entwicklung sind und inwiefern sie sich an medienübergreifende Tendenzen des Sprachwandels anschließen lassen.

\section{Internet-Kommunikation zwischen Mündlichkeit und Schriftlichkeit}

Wer die Unterschiede von geschriebener und gesprochener Sprache untersucht, merkt schnell, daß die mediale Realisierung von Sprache (phonisch vs. graphisch) nicht immer mit den Oppositionspaaren sprachlicher Merkmale korrespondiert, die man vorwissenschaftlich-intuitiv als typisch mündlich bzw. als typisch schriftsprachlich einordnen würde: „formell“ vs. „informell“, „,umgangssprachlich“" vs. „gehoben-schriftsprachlich“, „spontan gebildet“ vs. „,bewußt gestaltet“. Ein mündlicher Vortrag kann, sofern er auf einem Manuskript basiert, alle Merkmale elaborierter Schriftlichkeit aufweisen. Privatbriefe, Notizen können, obgleich schriftlich fixiert, vom Duktus her viele Merkmale der 
Mündlichkeit tragen. Viele dialogische Radio- und Fernsehsendungen inszenieren auf der Basis schriftlicher Vorlagen eine „sekundäre Mündlichkeit“, die sich aber in Herstellung und Resultat deutlich vom spontanen Gespräch von Angesicht zu Angesicht unterscheidet (vgl. Holly, 1992).

Die bei Koch und Oesterreicher (1994) erläuterte terminologische Differenzierung zwischen konzeptioneller und medialer Mündlichkeit bzw. Schriftlichkeit erlaubt es, die Frage nach dem Verhältnis von Sprech- und Schreibsprache präziser zu stellen:

- Mediale Mündlichkeit bzw. Schriftlichkeit bezieht sich auf das Medium, in dem Sprache realisiert wird. Dabei gibt es nur zwei Optionen: die phonetische Realisierung in gesprochener Sprache oder die graphische Realisierung in geschriebener Sprache. Es handelt sich also um eine dichotomische Abgrenzung.

- Konzeptionelle Mündlichkeit bzw. Schriftlichkeit meint dagegen den Duktus, die Modalität der Äußerungen, die man mit den oben genannte Gegensatzpaaren intuitiv in Verbindung bringt. Wichtig für das Konzept der Mündlichkeit ist die raum-zeitliche Nähe, aber auch die emotionale und soziale Nähe, die Bekanntheit und Vertrautheit der Gesprächspartner miteinander. Typisch für die Sprache der Nähe sind eine dialogisch konstituierte, offene Themenentwicklung sowie Emotionalität und Spontaneität. Das Konzept der Schriftlichkeit ist dagegen geprägt durch raumzeitliche, soziale und emotionale Distanz, bei der Produktion und Rezeption zeitlich auseinanderfallen und die Äußerungen von den Produzenten und der Produktionssituation abgelöst sind. Typisch für die Sprache der Distanz ist die monologische Themenorganisation ohne Kooperationsmöglichkeiten seitens der Rezipienten. Die Unterscheidung zwischen konzeptioneller Mündlichkeit und konzeptioneller Schriftlichkeit ist demnach eine graduelle: Nähe und Distanz bilden nach Koch und Oesterreicher (1994) zwei entgegengesetzte Endpunkte eines Kontinuums, auf dem sowohl medial mündliche Kommunikationsformen (z.B. Telefongespräch, wissenschaftlicher Vortrag etc.) als auch medial schriftliche Textsorten (Privatbrief, Zeitungsartikel etc.) angeordnet werden können. Wenn generell oder in Bezug auf Neue Medien von einer Vermündlichung der Schriftsprache die Rede ist, ist also immer die konzeptionelle Ebene gemeint, denn nur hier macht eine solche Aussage überhaupt Sinn. Orientierung an der Mündlichkeit bedeutet dann, daß die medial schriftlich fixierten Äußerungen Merkmale aufweisen, die als typisch für konzeptionelle Mündlichkeit gelten. Im folgenden möchte ich einige der bei Koch und Oesterreicher (1994) und bei Sieber (1998) angeführten Charakteristika konzeptioneller Mündlichkeit anführen, die mir für die Internet-Kommunikation besonders relevant erscheinen:

- In bezug auf die Lexik gibt es eine Präferenz für einfache und kurze Wörter, eine variationsarme Lexik, umgangssprachlich mar- 
kierte und dialektale Ausdrücke, viele und typisch sprechsprachliche Partikel und Interjektionen, die gehäufte Verwendung von Floskeln und sprachlichen Versatzstücken.

- Charakteristisch für die Syntax und den Textbau konzeptioneller Mündlichkeit sind der parataktische, reihende Satzbau, die wenig durchkomponierten Sätze, teilweise mit Satzbaufehlern, unklare Ganzsatzgrenzen, mit typisch sprechsprachlichen Konstruktionen, der inkonsequente Gebrauch von Kohäsionsmitteln und Gliederungssignalen, sowie eine freie, assoziative, dialogisch gesteuerte Themenentwicklung.

- Die kommunikative Grundhaltung der Mündlichkeit orientiert sich an dem Setting des alltäglichen Gesprächs von Angesicht zu Angesicht zwischen miteinander vertrauten Gesprächspartnern, die sich in der Sprecher- und Hörerrolle abwechseln. Typisch für dieses Setting sind kurze Planungszeiten bei der Produktion und kurze Verarbeitungszeiten für die Rezeption. Die Äußerungen werden meist spontan gebildet; die Themenentwicklung ist offen, wobei für die Teilnehmer in der Hörerrolle stets die Option der Rückfrage oder des Einspruchs besteht und die Teilnehmer in der Sprecherrolle mit sprachlichen und mimisch-gestischen Mitteln Feedback erhalten.

- Viele dieser Merkmale sind auch charakteristisch für schriftliche Texte im Internet, was sicherlich zurecht darauf zurückgeführt wird, daß die Netzkommunikation in besonderer Weise im Spannungsfeld zwischen Nähe und Distanz steht (Günther \& Wyss, 1996; Haase, Huber, Krumeich \& Rehm, 1997; Pansegau, 1997): Die Dienste des Internet befördern Dokumente über weite Entfernungen hinweg mit großer Schnelligkeit. Die räumliche Distanz der Kommunikationspartner wird also kompensiert durch die zeitliche Nähe, die durch den raschen Wechsel (bis hin zur Simultankommunikation im Chat, s.u.) entsteht. Die metaphorisch konstituierte räumliche Nähe - man befindet sich in demselben Chatraum, man wird als Besucher auf einer Homepage willkommen geheißen, man gehört zur „Netzgemeinde“ - schafft zusätzlich emotionale und soziale Nähe. Allerdings sind die Merkmale konzeptioneller Mündlichkeit in den verschiedenen Diensten und Kommunikationsformen des Internet in sehr unterschiedlicher Weise ausgeprägt. Im folgenden Abschnitt werden die Gemeinsamkeiten und Unterschiede erläutert, die in dieser Hinsicht zwischen Email-, Newsund Chatkommunikation bestehen. 


\section{Dienste und Kommunikationsformen im Internet und ihre sprachlichen Merkmale}

Das Internet läßt sich aus technischer Sicht definieren als Netz von Computernetzen, die über die Protokollsprache TCP/IP Daten austauschen. Aus funktionaler Sicht besteht es aus einer Reihe von Diensten, die von Nutzern in Anspruch genommen werden können; diese lassen sich weiter differenzieren in Kommunikationsdienste und Informationsdienste (vgl. Kuhlen, 1995). Dienste sind im technischen Sinne definiert durch ein für sie charakteristisches Protokoll (z.B. SMTP für Email, HTTP für das WWW). Während bis in die 90-er-Jahre hinein für jeden Dienst je ein spezielles Programm benötigt wurde, hat das WWW zur Entwicklung multifunktionaler Anwendungsprogramme (z.B. die Netscape-Programmfamilie) geführt. Diese verbergen die technische Komplexität hinter graphischen Oberflächen und konzeptualisieren das Internet als Kommunikationsraum, den die Nutzer betreten ${ }^{2}$ und an dem sie in verschiedener Weise partizipieren können. Um folgende Partipizationsmöglichkeiten wird es in diesem Beitrag gehen:

- Die Möglichkeit, Briefe an Adressaten und Adressatengruppen zu verschicken (Email).

- Die Möglichkeit, sich an der öffentlichen Diskussion über ein Thema mit eigenen Beiträgen zu beteiligen bzw. die Beiträge anderer öffentlich zu kommentieren (Newsgroups/Foren).

- Die Möglichkeit, zeitgleich mit anderen getippte Gespräche zu führen (Chat).

Diese unterscheiden sich durch Merkmale des kommunikativen Settings (,synchron“ vs. ,asynchron“, „öffentlich“ vs. „privat/institutionell“ etc., vgl. Lenke \& Schmitz, 1995) sowie durch die speziellen Funktionen, die sie für die Kommunikation bereitstellen. Um den Zusammenhang zwischen kommunikativem Setting, Softwarefunktionen und den sprachlichen Merkmalen konzeptioneller Mündlichkeit wird es im folgenden gehen.

\subsection{Email-Kommunikation}

Einer der ältesten und bekanntesten Dienste des Internet ist die Email. Als Email bezeichnet man im Deutschen sowohl den Postdienst als auch die Schriftstücke, die über diesen Dienst befördert werden. Im Vergleich mit der als ,snail-mail“ bezeichneten ,gelben Post“ ist Email jedoch nicht nur erheblich schneller, sondern auch vielseitiger und kostengünstiger, vor allem, wenn

2 Vgl. z.B. den Ausruf Boris Beckers, ,ich bin drin“, in einem aktuellen Fernsehwerbespot. 
ein Computer mit Internet-Anschluß schon vorhanden ist und häufig Briefe an vielköpfige Adressatengruppen versandt werden müssen. Die Schnelligkeit des Briefwechsels und die komfortablen Funktionen zum Beantworten, Verteilen und Weiterleiten von Briefen machen Email nicht nur Alternative zum traditionellen Brief, sondern auch und vor allem zu Fax und Telefon (vgl. Pansegau, 1997; Günther \& Wyss, 1996; speziell zum Vergleich von Email- und Faxkommunikation auch Quasthoff, 1997). Email ermöglicht es beispielsweise, innerhalb eines Vormittags eine Terminabsprache zwischen mehreren, an verschiedenen Orten arbeitenden Menschen zu treffen, ohne diese durch lästiges Hin- und Hertelefonieren zu stören und gilt deshalb in Umfragen auch als der Dienst im Internet, auf den die Nutzer am wenigsten verzichten möchten (vgl. die Rubrik ,indispensable“ der 10. GVUNutzerumfrage, GVU-10, 1998).

\section{Abbildung 1: Terminaushandlung per Email}

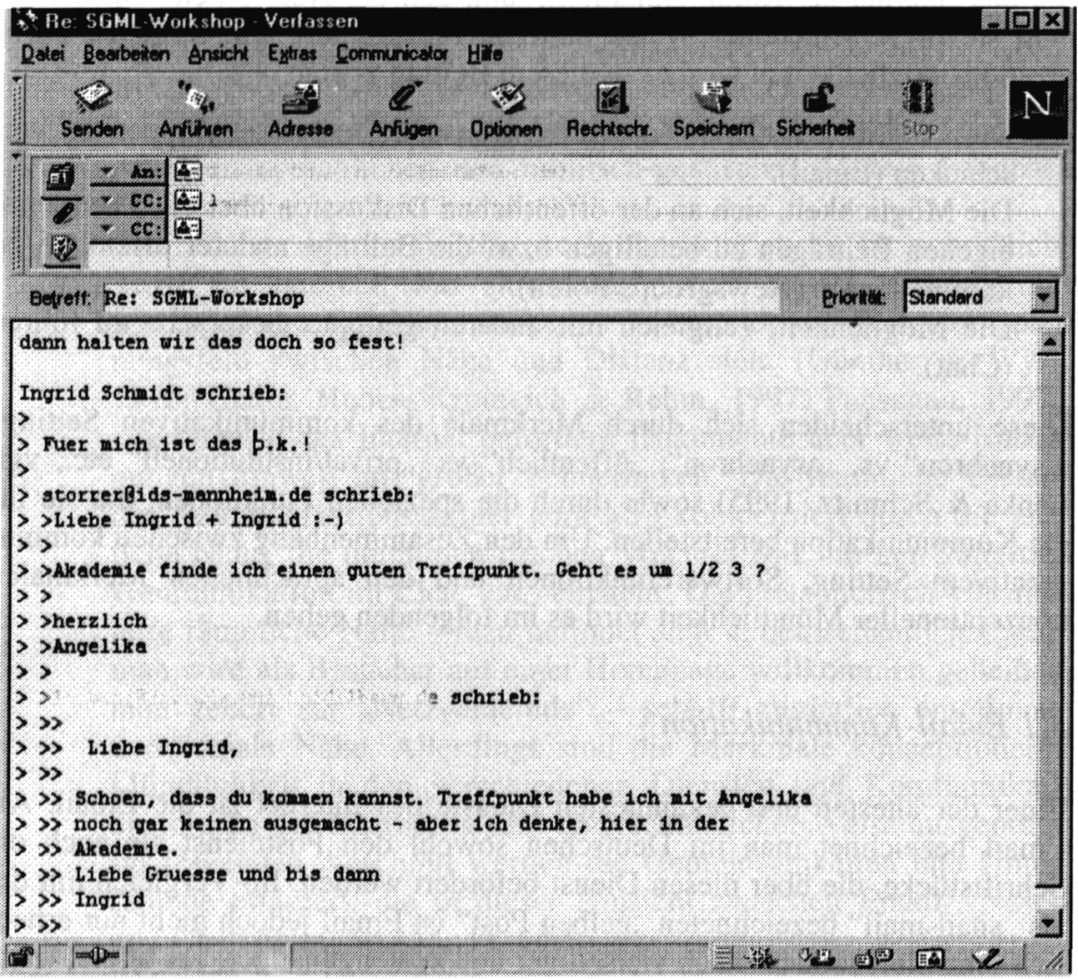

Momentan haben die digitalen Briefe im Vergleich zu den Papierbriefen der gelben Post noch einige Nachteile: Sie können nicht handschriftlich verfaßt 
und nicht mit einer rechtsgültigen Unterschrift versehen werden. Sie unterliegen nicht dem Briefgeheimnis und sollten deshalb im Bedarfsfall verschlüsselt werden. An der Verbesserung dieser Defizite wird jedoch gearbeitet. Diese Nachteile werden aber aufgewogen durch Softwarefunktionen, die es ermöglichen, den zeitlichen Aufwand für die private, innerbetriebliche und öffentliche Briefkommunikation zu verringern:

- Die „Reply“-Funktion generiert einen E-Brief mit der E-Post-Adresse des Absenders. Dabei kann eine Kopie des erhaltenen Brieftextes angelegt werden, die durch bestimmte Zeichen, meist ein ,>" am Zeilenanfang markiert ist; man spricht hier von „Zitieren“ bzw. „Zitaten“. Zitate sind zeitsparend, da die im Brief angesprochenen Anliegen nicht mehr reformuliert werden müssen, sondern direkt beantwortet werden können. Da die Zitierfunktion rekursiv anwendbar ist, kann die wechselseitige Aushandlung eines Themas über mehrere E-Briefe hinweg festgehalten werden (vgl. Abbildung 1).

- Die „Forward“-Funktion ermöglicht es, einen E-Brief unkompliziert an eine andere E-Post-Adresse weiterzuleiten, wobei dieser vorher gekürzt, verändert oder kommentiert werden kann.

- Die „Signature“-Funktion fügt automatisch an das Ende jedes E-Briefs einen Textbaustein, der von einer einfachen Adressenangabe bis hin zu aufwendig gestalteten ASCII-Gemälden (vgl. Wyss, 1999) alles enthalten kann.

Neben privatem und offiziellem Briefverkehr organisiert der elektronische Postdienst auch sog. „Mailinglisten“. Es handelt sich um Postverteiler, deren Abonnenten Neuigkeiten, Diskussionsvorschläge etc., die von anderen Abonnenten verfaßt werden, zugestellt bekommen. Wer die verteilten Beiträge beantworten oder kommentieren möchte, schickt eine Email entweder direkt an den Autor des Beitrags oder wieder an den Postverteiler, der die Antwort dann an alle Abonnenten verteilt.

Da Email, wie auch die gelbe Post, für sehr viele Kommunikationszwecke genutzt werden kann, decken die mit ihr beförderten Schriftstücke ein sehr breites Spektrum zwischen konzeptioneller Mündlichkeit und Schriftlichkeit ab:

- Wer sich über Email auf eine Stelle bewirbt oder mit Kunden oder Vorgesetzten kommuniziert, orientiert sich sicher weiter an den Textmustern des traditionellen Briefverkehrs.

- Wer Email als Alternative zu Fax oder Telefon wählt, nutzt meist die Zitier- und Kopierfunktionen und wählt einen stark an der konzeptionellen Mündlichkeit orientierten Formulierungsstil.

Gerade der Vergleich verschiedener Email-Schriftstücke zeigt, daß natürlich auch im neuen Medium die Funktion die Form dominiert, anders gesagt: Die Textgestaltung richtet sich in erster Linie nach dem kommunikativen Hand- 
lungsziel, das mit der Textproduktion verfolgt wird. Dies zeigt u.a. die Erfahrung, die die WWW-Redaktion des Hypertextes zur neuen deutschen Rechtschreibung am IDS (http://www.ids-mannheim.de/grammis/reform.html) gesammelt hat. Die Emails von Site-Betreibern, die darum bitten, den Hypertext in ihrem Intranet kopieren (spiegeln) zu dürfen, besitzen mehrheitlich höfliche Anreden und Schlußformeln; sie enthalten so gut wie keine Tippfehler und sind vom Textaufbau und von der Elaboriertheit in Wortwahl und Satzbau mit entsprechenden ,traditionellen“ Briefen vergleichbar (vgl. Abbildung 2).

Abbildung 2: Anfrage wegen einer Kopiergenehmigung,

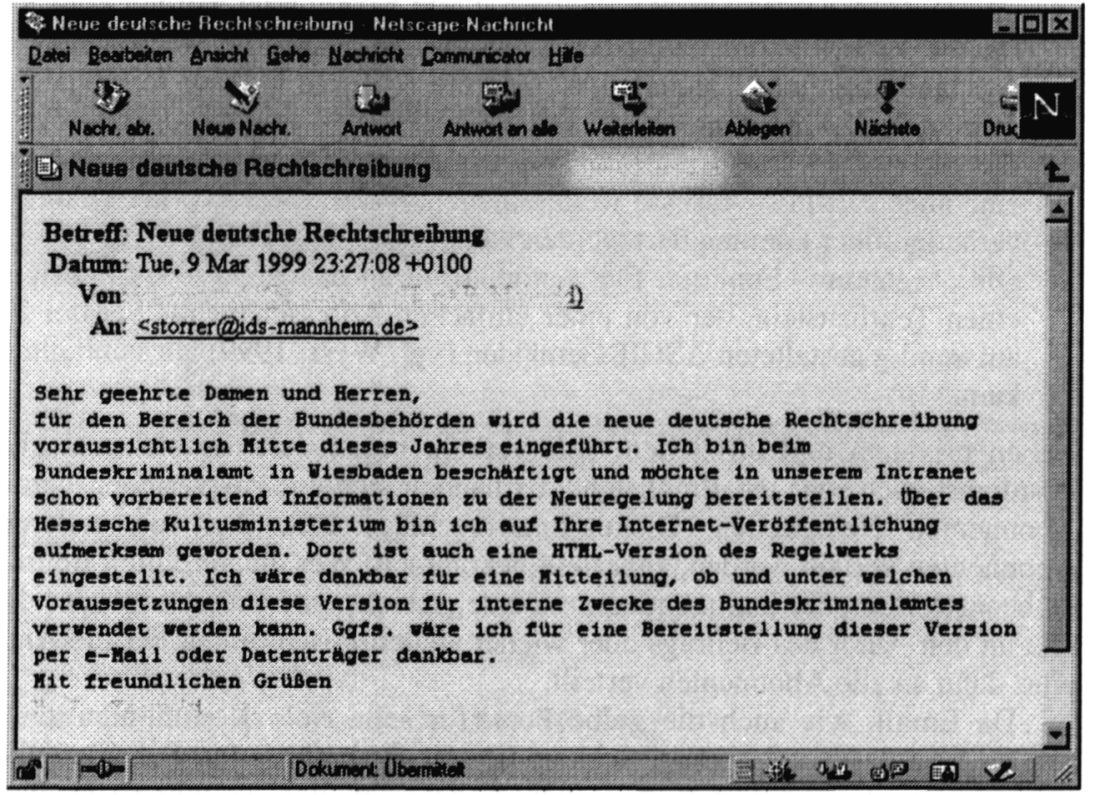

Dasselbe gilt für die Schreiben, in denen der Redaktion für die Bereitstellung und Genehmigung des Spiegels gedankt wird. Der konzeptionell mündliche Stil findet sich eher in privaten Emails, in Mailinglisten und Foren (s.u.) und in institutionellen Kontexten, in denen Emails als Alternative zu Fax und Telefon genutzt werden, wie bei der Terminabsprache in Abbildung 1. Die Briefwechsel werden zu „Turn"-Wechseln, die nach dialogischem Muster z.B. als Frage-Antwort-Sequenzen, als Bitte-Danke-Sequenzen, als Behauptung-Kommentar-Sequenzen - organisiert sind. $\mathrm{Da} \beta$ solche Sequenzen mehrmals am selben Tag hin- und hergeschickt werden können, ist in vielen Fällen sicher auch ein Grund für die Weglassung der Anrede bei Emails. Wenn 
an einem Tag schon ein Briefwechsel gelaufen ist, ist eine nochmalige Begrüßung eigentlich kommunikativ unangemessen. Email-Nutzer, die dennoch nicht darauf verzichten möchten, behelfen sich mit medienspezifischen Grußformeln wie ,re-hi“ oder ,nochmal gruesse“ ${ }^{33}$.

\subsection{Kommunikation in Nachrichtenforen}

Ein ebenfalls bereits ,älterer“ Dienst ist das UseNet, das 1979 an der Universität von Duke in North Carolina entstand und sich in der Folge rasch ausbreitete. Das UseNet organisiert inzwischen mehrere tausend Foren (,newsgroups") zu verschiedensten Lebensbereichen und Themen, teilweise nur mit lokaler Verbreitung, denn Ende der 80er Jahre wurden die englischsprachigen Gruppen allmählich um nationale Foren ergänzt, die in den jeweiligen Landessprachen diskutieren (vgl. Hoffmann, 1997). Die Foren unterscheiden sich in zwei Punkten von den oben erwähnten Emailverteilern:

- Die Verbreitung läuft nicht nach dem Push- sondern nach dem Pull-Verfahren: Die Beiträge des UseNet werden nicht an einen festgelegten, identifizierbaren Abonnentenkreis geschickt, sondern können von allen Interessierten abgerufen werden. Dafür sorgt ein weltweiter Verbund von sog. „Newsservern“, die Beiträge austauschen und diese zum Abruf bereitstellen. Die Nutzer können diese Beiträge mit Hilfe sog. „Newsreader" lesen, beantworten und um eigene Beiträge ergänzen; solche Newsreader sind inzwischen in viele WWW-Browser integriert (z.B. in den Netscape Communicator). Manche Foren, wie das in Abbildung 3 gezeigte Forum ,de.sci.paedagogik“, sind inzwischen direkt über das WWW zugänglich, d.h. sie kodieren ihre Beiträge auch in HTML.

- Wie kein anderer Dienst versteht sich das UseNet nicht vornehmlich als neue Kommunikationstechnik, sondern als Gemeinschaft mit eigenständigen Verhaltensregeln und Normen. „Das UseNet ist kein Netz, sondern eine Gemeinschaft" heißt es explizit in einem regelmäßig verbreiteten Anleitungstext zum richtigen Verhalten im UseNet (Partl o.J.), das Neulinge vor Fehlverhalten und Fettnäpfchen bewahren möchte. Die Beiträge des UseNet zeigen am deutlichsten die typischen Merkmale des kommunikativ-sozialen Stil im Internet (vgl. Abschnitt 4), der geprägt ist von

3 Hess-Lüttich weist darauf hin, daß der Briefwechsel schon von jeher als dialogisch, als „phasenversetztes Gespräch" als „amicorum conloquia absentium“ verstanden worden ist (Hess-Lüttich 1997, S. 226). Die potentielle Möglichkeit der Verkürzung der „Phasen“, die zwischen der Verschickung des Briefes und dem Erhalt einer Antwort liegen (u.U. nur Minuten), sowie die Art der wechselseitigen Bezugnahme (über Zitate statt über Reformulierung) rechtfertigen es m.E. jedoch, Emails eine qualitativ anders geartete Dialogizität zuzusprechen, d.h., sie auf dem Spektrum zwischen kommunikativer Mündlichkeit und Schriftlichkeit an anderer Stelle einzuordnen. 
Wertvorstellungen wie Selbstorganisation, Meinungsfreiheit und kostenfreiem Austausch von Wissen und Ressourcen, die noch aus den Gründerjahren des Netzes stammen und im UseNet weiterhin Gültigkeit beanspruchen.

- Viele Foren verfügen über eine langjährige gemeinsame Kommunikationsgeschichte, die über eine spezifische Textsorte tradiert wird, die FAQ (Frequently Asked Questions). Unter FAQ werden Fragen und Themen aufgeführt, die innerhalb der Gruppe bereits mehrfach beantwortet oder lang und breit ausdiskutiert wurden und mit denen man sich nicht noch einmal beschäftigen möchte. Einsteigern wird ans Herz gelegt, zunächst die FAQ des Forums zu lesen, bevor sie einen eigenen Beitrag veröffentlichen.

Die Beiträge in den Foren machen ausgiebigen Gebrauch von der Zitierfunktion. Während in der Email-Kommunikation die Briefe jedoch häufig hintereinander kopiert sind, wobei das aktuelle Schreiben meist zuoberst erscheint, ist es für Beiträge in Foren typisch, nur die Textstellen von Beiträgen zu zitieren, zu denen man einen Kommentar, einen Hinweis, eine weitere Frage hinzufügen möchte; der eigene Beitrag erscheint dann unterhalb der Zitate (vgl. Abbildung 3). Durch wechselseitige Kommentierung von initialen Beiträgen entstehen Diskussionsstränge, sog. ,threads“, die durch die Zitierkonventionen für Geübte in ihrem Verlauf leicht nachvollziehbar sind. In dem in Abbildung 3 gezeigten Beispiel wird eine im Initialbeitrag gemachte Aussage von Autor A (erkennbar an drei Zitatzeichen) von Autor B durch die mit zwei Zitatzeichen gekennzeichneten Fragen in Zweifel gezogen. Autor A wechselt mit der Gegenfrage „Was soll das?“ und weiteren Anmerkungen (gekennzeichnet mit einfachen Zitatzeichen) auf die metakommunikative Ebene, auf der Autor B wacker mithält. Das Beispiel zeigt einen Verlauf, den Diskussionen im UseNet häufig nehmen: Die Abkehr von der Sachdiskussion zur Metadiskussion, die wiederum schnell ins Persönlich-Beleidigende, ins sog. „Flaming“, abdriftet. Das schnelle Aufflackern von Streitlust geht vermutlich auf zwei Faktoren zurück:

- Auf die Unverbindlichkeit und Anonymität des kommunikativen Settings, aus dem man sich jederzeit zurückziehen kann und damit freibleibt von den üblichen sozialen Sanktionen, mit denen man im wirklichen Leben zu rechnen hat. Selbst wenn man von einer Gruppe weitgehend ignoriert wird - wozu das Internet durch „kill-files“ oder „Bozo“-Filter auch technische Möglichkeiten bietet (vgl. Waldenberger \& Storrer, 1998, S. 69) - kann man versuchen, mit neuer Identität weiter zu machen, sollte dies durchschaut werden, liegt die nächste Gruppe nur einen Mausklick weit entfernt.

- Aus der Stimm- und Gesichtslosigkeit der Kommunikation, d.h. der fehlenden Notwendigkeit, dem Gegner beim verbalen Angriff ins Gesicht schauen zu müssen. Dies scheint dazu zu führen, daß schnell vergessen wird, daß hinter den am Bildschirm erscheinenden Beiträgen Menschen stecken, die ihr Gesicht wahren möchten. 
Die wichtigste Regel der Netikette lautet deshalb: „Vergessen Sie nie, daß Sie es mit Menschen zu tun haben!“" (,Remember the human!"). Allerdings gehört Streitbarkeit durchaus zum kommunikativen sozialen Stil der Netzgemeinde; deshalb wird Flaming toleriert, solange die Streithähne mit ihren gegenseitigen Angriffen nicht die Kommunikation der Gruppe behindern. Langwierige und ausufernde Streitereien, sog. „flame-wars“, sind jedoch unerwünscht (vgl. Storrer \& Waldenberger, 1998, S. 74).

Abbildung 3: Auseinandersetzung in einem Forum

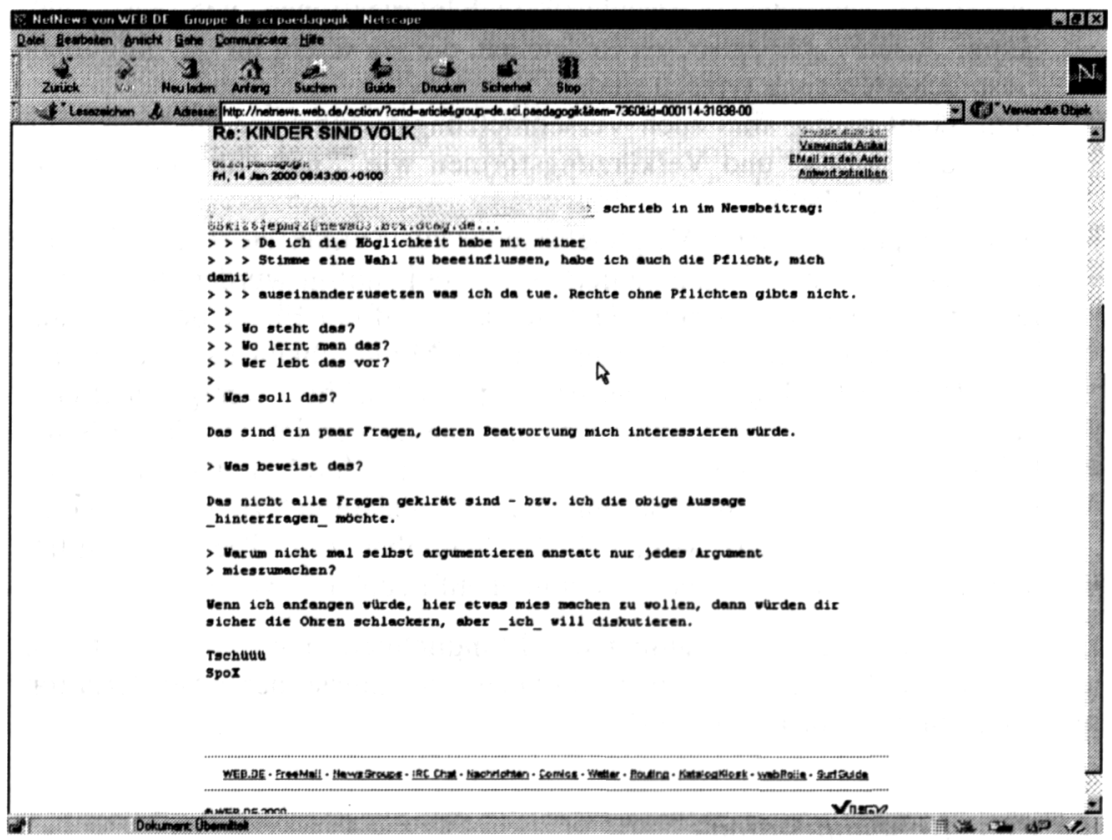

Da die UseNet-Beiträge öffentlich und frei verfügbar sind, können sie auf relativ einfache Weise archiviert und mit automatischen Verfahren quantitativ ausgewertet werden. Am Seminar für Sprachwissenschaft der Universität Tübingen wurde im Rahmen des ELWIS-Projekts (Storrer, Feldweg \& Hinrichs, 1993) ein umfangreiches Corpus mit Beiträgen aus deutscher Nachrichtenforen aufgebaut und durch automatische Frequenzanalysen verglichen mit einem Zeitungscorpus der Frankfurter Allgemeinen Zeitung ${ }^{4}$. Die Ergebnisse bestätigen den Eindruck, daß die in den Foren veröffentlichen Beiträge

4 Das Newscorpus umfasste den Jahrgang 1993 mit ca. 43 Millionen Wortformen, das Zeitungscorpus den Zeitraum von Juli 1992 bis März 1993 mit ca. 35 Millionen Wortformen (vgl. Feldweg, Kibiger \& Thielen, 1995). 
starke Züge mündlicher Alltagskommunikation aufweisen. Insbesondere die in Abschnitt 2 genannten lexikalischen Merkmale für konzeptionelle Mündlichkeit ließen sich durch die quantitativen Verfahren deutlich nachweisen (vgl. Feldweg, Kibiger \& Thielen, 1995):

- Umgangssprachlich markierte und dialektal eingefärbte Ausdrücke treten gehäuft auf; dies zeigt sich in einer Untersuchung schweizerischer Emails noch deutlicher (Günther \&Wyss, 1996).

- Nachgewiesen wurde auch ein hoher Anteil an Modal- und Abtönungspartikeln wie ,schon“, „wohl“, „,mal“, ,,also“, „eigentlich“, Adverbien wie „,wirklich“, ,übrigens“, „,irgendwie“" und Interjektionen, wie „na“, „äh“, „,hmm“, ,,ach“, „,oh“, also von Redeteilen, die vor allem für die mündliche Gesprächssteuerung typisch sind.

- Signifikant häufig sind auch Verschmelzungsformen wie „haste“, „,willste“ oder „gibt's“" und Verkürzungsformen wie „,ne“ (für „eine“) oder „hab“" (für „habe“).

- Eine computergestützte Vergleichsstudie zur Syntax steht derzeit noch aus, da dafür syntaktisch annotierte Corpora benötigt würden. Sicher wäre aber niemand überrascht, wenn sich auch auf der Ebene des Satz- und Textbaus viele Merkmale konzeptioneller Mündlichkeit nachweisen lieBen. Typisch für Forenbeiträge ist ein parataktischer, wenig integrierter Satzbau. Häufig werden Sätze lose aneinander gereiht und durch Semikolon getrennt. Typisch ist es auch, einen Satz durch drei Auslassungspunkte statt durch einen Satzendepunkt ,auslaufen“ zu lassen, womit vermutlich signalisiert wird, daß der Schreiber zum Thema eigentlich mehr zu sagen hat, nun aber erst einmal auf Feedback wartet.

Die kommunikative Grundhaltung der Mündlichkeit manifestiert sich u.a. auch im vertraulichen ,du“ und in saloppen, umgangssprachlichen Grußformeln wie „moin“, „tach“, „tschüs“, „,ciao“ oder „hi“.

\subsection{Chat-Kommunikation}

Die auf den ersten und wohl auch auf den zweiten Blick spektakulärsten sprachlichen Eigentümlichkeiten zeigen sich in der Chat-Kommunikation. Beim Chat können sich Teilnehmer in Kanäle einwählen, die metaphorisch als „Räume“ bezeichnet werden und dort simultan miteinander ein $\mathrm{Ge}$ spräch über Tastatur und Bildschirm führen. Der Schnappschuß in Abbildung 4 zeigt einen Ausschnitt aus dem SpaceChat, der von der WWW-Site des Uni-Magazins ,unicum" angeboten wird ${ }^{5}$. Ein solcher statischer Chat-

5 Http://www.unicum.de/frames/chat.htm. Der „SpaceChat“ liegt auch der Untersuchung in Beißwenger (1999) zugrunde; Funktionsweise und Eigentümlichkeiten sind darin ausführlich beschrieben. 
Ausschnitt erweckt für Außenstehende zunächst den Eindruck, die Teilnehmer seien in ihrer Sprachentwicklung bei Zwei- und Dreiwortsätzen stehen geblieben und redeten munter aneinander vorbei. Wer jedoch selbst an einem Chat teilnimmt, merkt schnell, daß das Chatten eine gute Konzentrationsfähigkeit, eine schnelle Reaktion und ein hohes Ausmaß an Phantasie und Vorstellungsvermögen erfordert. Da die Beiträge in rascher Abfolge über den Bildschirm laufen, ist es gerade für Ungeübte nicht einfach, dem „Gesprächsverlauf“" zu folgen und gleichzeitig mit eigenen Beiträgen zu reagieren.

Chatkanäle sind eine mediengeschichtliche Novität: Sie erlauben die simultane Kommunikation über weite räumliche Distanz hinweg mittels geschriebener Sprache in getippter Form (vgl. Lenke \& Schmitz, 1995; Beißwenger, 1999). Die mediale Schriftlichkeit unterscheidet den Chat von den medial mündlich organisierten Medien „Telefon“ und „Hobbyfunk“ sowie von der Kommunikationsform „Videokonferenz“, die dem Chat vom kommunikativen Setting her am nächsten sind. Durch seine Synchronität ist der Chat natürlich noch stärker der konzeptionellen Mündlichkeit verpflichtet, als die asynchronen Kommunikationsformen Email und News. Entsprechend weisen die Mitschnitte viele Merkmale medial gesprochener Sprache auf: Die einzelnen Beiträge (,turns") sind in den seltensten Fällen syntaktisch vollständig. Interpunktionszeichen sind zwar vorhanden, allerdings fehlt im Normalfall ein Satzendepunkt. Die vorhandenen Interpunktionszeichen sind nur selten nach den für Schrifttexte gängigen Konventionen gesetzt, sondern übernehmen vornehmlich expressive Funktion.

Die Metapher des Chatraums, den die Chatter betreten und verlassen können, knüpft ebenfalls an das prototypische Setting für konzeptionelle Mündlichkeit an: An das Gespräch von Angesicht zu Angesicht (face-toface) zwischen Menschen, die sich kennen und sich zur selben Zeit im selben Raum befinden. Dennoch kann man sich des Eindrucks nicht erwehren, $\mathrm{da}$ die hier entstehenden „Gespräche“ eigentlich doch wenig mit den $\mathrm{Ge}$ sprächen zu tun haben, die wir im Alltag führen. Dies liegt an den Unterschieden, die zwischen dem kommunikativen Setting der Chat-Kommunikation und dem Setting der Face-to-Face-Kommunikation bestehen: 
Abbildung 4: Ein Auszug aus dem „SpaceChat“

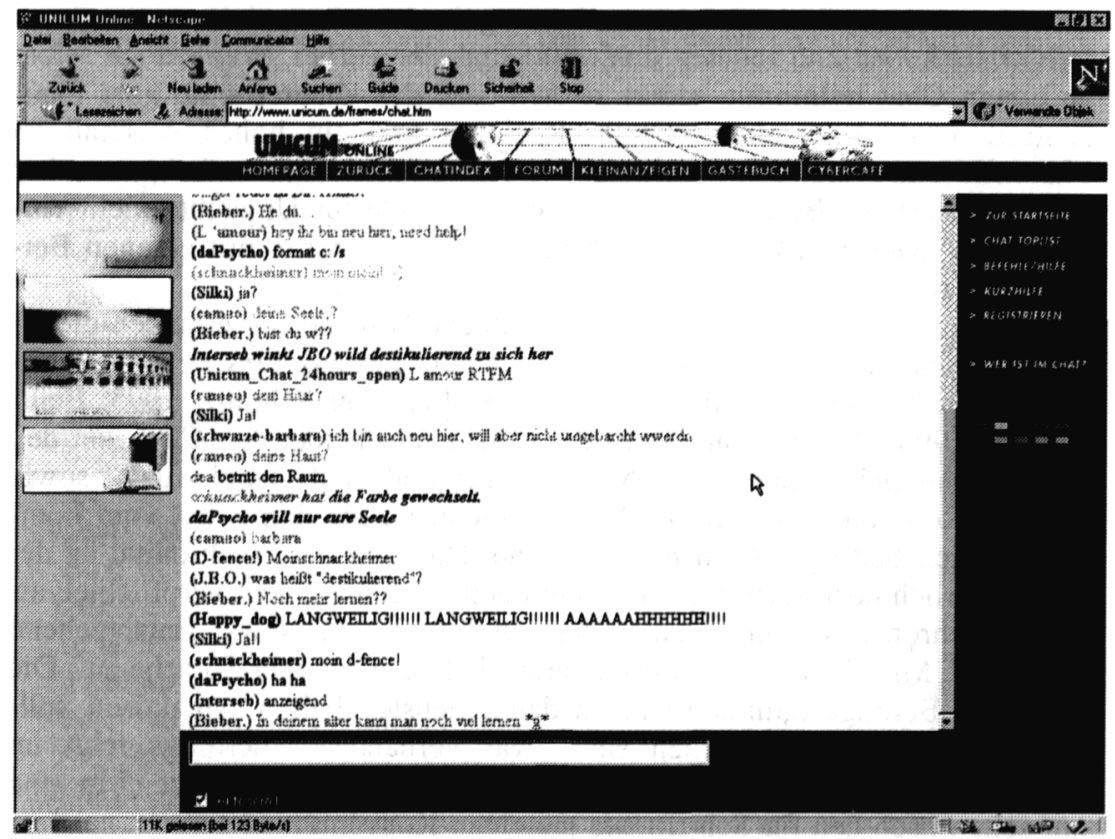

- Im Gespräch von Angesicht zu Angesicht verfügen die Beteiligten über einen gemeinsamen Wahrnehmungsraum, auf den sprachlich und gestisch gezeigt und bezug genommen werden kann. Im schriftbasierten Chat hingegen ist das gemeinsame Wahrnehmungsfeld auf den Bildschirm beschränkt, auf dem die Gesprächsbeiträge in der Reihenfolge ihres Eintreffens angezeigt werden. Ist der Bildschirm voll, werden die ,alten“ Beiträge nach oben geschoben, gelangen also aus dem Sichtfeld. Wenn viele Chatter im selben Raum rege miteinander kommunizieren, kann man deshalb schnell die Übersicht über die „Anwesenden“ verlieren. Viele Turns dienen dann vornehmlich dazu, sich erneut „sichtbar“ zu machen und Kommunikationsbereitschaft zu signalisieren (vgl. Beißwenger, 1999); diese Funktion haben in Abbildung 4 vermutlich die wiederholten Fragen bzw. Ausrufe von „Silki“ und der Aufschrei von „Happydog" (LANGWEILIG!!!!! ...).

- Im Gespräch von Angesicht zu Angesicht läuft die Verständigung simultan über mehrere Wahrnehmungskanäle (auditiv, visuell, olfaktorisch), die sich wechselseitig beeinflussen. Parasprachliche Zeichensysteme wie Mimik und Gestik sowie prosodische Merkmale wie Intonation, Akzent, Tonhöhe spielen für die Interpretation des Gesagten eine wesentliche Rolle. Chat-Kommunikation hingegen beschränkt sich auf den visuellen Kanal, 
auf die Anzeige schriftlicher Turns am Bildschirm. Allerdings gibt es verschiedene Konventionen, um die konzeptionell mündlich geprägte Kommunikationsform um Elemente der medialen Mündlichkeit anzureichern; diese sind in der Literatur zur Chatkommunikation bereits beschrieben und kategorisiert (z.B. Lenke \& Schmitz, 1995; Haase, Huber, Krumeich \& Rehm, 1997; Beißwenger, 1999; Runkehl, Schlobinski \& Siever, 1998). Zu ihnen gehören die sog. Emotikons (vgl. 4.2), die zur emotionalen Kommentierung von Turns (z.B. freundliche Grundhaltung, Gute Laune, Ärger, Enttäuschung) eingesetzt werden können. Andere Mittel zum Ausdruck von Gefühlszuständen und Handlungen nehmen Anleihen an comicsprachliche Wortbildungsmuster, z.B. *heul*, *freu*, *knuddel*; das „*" ${ }^{*}$ “ am Ende des letzte Turns in Abbildung 4 ist eine bereits konventionalisierte Kurzform für ,grins* bzw. *grin*. Diese Bildungen und Abkürzungen, die auch vereinzelt in der Email- und News-Kommunikation vorkommen, sind in der Chat-Kommunikation sehr häufig und in verschiedensten Formen und Funktionen verwendet (vgl. Beißwenger, 1999).

- Beim Gespräch von Angesicht zu Angesicht gibt es Prinzipien und Konventionen, nach denen die Vergabe des Rederechts und der Sprecherwechsel (das Turntaking) organisiert werden; diese determinieren letztlich die sequentielle Abfolge der Redebeiträge (vgl. Zifonun, Hoffmann, Strecker, 1997). In der Chat-Kommunikation dagegen ist das Turntaking rein technisch bestimmt. Es erfolgt, wie Wichert (1991, S. 78) es treffend formuliert hat, nach dem „Mühlen-Prinzip: wer zuerst kommt, mahlt zuerst." Das heißt, die Gesprächsbeiträge erscheinen in der Reihenfolge am Bildschirm, in der sie beim Chat-Server, der Software, die den Chat organisiert, eintreffen. Dieser Unterschied hat eine Reihe von Konsequenzen (vgl. auch Lenke \& Schmitz, 1995; Beißwenger, 1999):

- Durch die technisierte Turnorganisation können sich im Chat keine Überlappungen ergeben, wie sie im Alltagsgespräch für Übergangspunkte beim Sprecherwechsel typisch sind.

- Auch das Konzept des Unterbrechens macht keinen Sinn, denn es setzt voraus, daß Gespräche so organisiert sind, daß meist nur eine Person die Sprecherrolle innehat, während die anderen schweigen und einen geeigneten Moment für die Ergreifung des Rederechts abwarten. Im Chat hat jeder stets das Recht, einen neuen getippten Beitrag abzuschicken. Es muß deshalb ständig gleichzeitig gelesen und geschrieben werden, was gerade für Neulinge recht gewöhnungsbedürftig ist. Dies dürfte einer der Gründe dafür sein, daß nur sehr kurze Turns formuliert werden bzw. längere Beiträge auf verschiedene Turns verteilt werden, wie es der Teilnehmer „cameo" in Beispiel 4 mit der an die „schwarze Barbara“ gerichteten Frage tut, was sich hinter dem ,schwarz" verbirgt.

- Trotz der Simultanität des Chat - die Teilnehmer sitzen zeitgleich am Computer - fallen Produktions- und Äußerungsakt zeitlich aus- 
einander. Erst wenn die Return-Taste betätigt wird, wird der Beitrag für die Rezipienten sichtbar. Die eigenen Redebeiträge müssen also in Unkenntnis darüber produziert werden; wer zur selben Zeit noch an einem Turn tippt. Dadurch kann es zu Überschneidungen kommen, d.h. eine Reaktion auf einen Turn wird erst dann sichtbar, nachdem der Produzent dieses Turns schon einen weiteren Turn abschickt hat.

- Die Sequenzierung nach dem Mühlen-Prinzip erschwert es erheblich, die kommunikativen Züge hinter den Turns zu erkennen. Je mehr Teilnehmer sich in einem Chatraum befinden, desto schwieriger wird es, nachzuvollziehen, wer hier überhaupt mit wem kommuniziert. Technik soll die Übersichtlichkeit verbessern: Im SpaceChat bekommen beispielsweise alle Teilnehmer eine charakteristische Farbe zugeordnet, die sie im übrigen, wie der Teilnehmer ,Schnackheimer“ in Beispiel 4, auch wechseln können. Dies hilft den Chattern, die Aufmerksamkeit auf bestimmte Teilnehmer zu konzentrieren. Zusätzlich gibt es die Möglichkeit, die Gesprächsbeiträge an bestimmte Teilnehmer im Chatraum zu adressieren. Hierfür haben sich bestimmte Muster und Konventionen herausgebildet: In Beispiel 1 ist die Aufforderung „RTFM“6 an den Teilnehmer ,l'amour“ adressiert; es handelt sich um eine nicht gerade nette Antwort auf dessen Hilfegesuch „hey ihr bin neu hier, need help!“.

Gerade wegen der Besonderheiten beim Turntaking darf bezweifelt werden, ob sich das Chatten als Kommunikationsform wirklich für den problem- und themenorientierten Gedankenaustausch innerhalb größerer Gruppe eignet, z.B. für Online-Seminare im Hochschulbereich, für Arbeitsbesprechungen und Diskussionen von räumlich verteilten Arbeitsgruppen. Vermutlich werden sich hier andere Formen, z.B. Videokonferenzen, durchsetzen. Der Chat hat andere Reize: Beißwenger (1999) zeigt auf der Basis eines umfangreichen Corpus von Mitschnitten aus dem SpaceChat, daß es beim Chat weniger um Gedankenaustausch als um die ,kooperative Inszenierung von Welt im Spiel mit der Virtualität" geht (Beißwenger, 1999, S. 166). Dies beginnt bei der Wahl der Namen für die Räume, in denen kommuniziert wird, und des Namens, mit dem man den Chatraum „betritt“. Es ist im übrigen durchaus möglich, mit mehreren Namen denselben Chatraum zu betreten und die beiden „virtuellen Identitäten" miteinander flirten oder streiten zu lassen.

Die Nutzung des Chat als schriftlich konstruiertes Online-Improvisationstheater wird durch Funktionen unterstützt, die es ermöglichen, die Äußerungen um Handlungsbeschreibungen zu ergänzen. Einige davon werden als

6 Das Akronym löst sich auf in „Read that fucking manual” und bezieht sich auf eine der dienstübergreifenden Regeln der „Netikette“: Einsteiger in eine neue Kommunikationsform sollen die Dokumentation und die Hilfetexte lesen, bevor sie ältere „Netzbürger“ um Hilfe bitten (vgl. Partl, o.J.). 
sprachliche Versatzstücke automatisch vom System generiert, um bestimmte Aktionen der Teilnehmer anzuzeigen, z.B. das Betreten und Verlassen eines Raumes (in Abbildung 4: „dea betritt den Raum“). Die Teilnehmer können sich aber auch selbst Handlungen zuschreiben, die dann vom System aus einer Außenperspektive verbalisiert werden (in Abbildung 4 ,Interseb winkt JBO wild destikulierend zu sich her“ bzw. „daPsycho will nur eure Seele“). Dies erweitert das kommunikative Spektrum um beschreibende und erzählerische Momente und schafft überhaupt erst die Voraussetzung für das Spiel mit Identitäten, Masken und Rollen, das für viele Chats charakteristisch ist.

\section{Dienstübergreifende sprachliche Merkmale in der Internet-Kommunikation}

Neben den im vorigen Abschnitt besprochenen Merkmalen, die unmittelbar auf technische Funktionen und Gepflogenheiten bestimmter Dienste bezogen sind, gibt es sprachliche Auffälligkeiten, die in allen Diensten in mehr oder weniger ausgeprägter Weise vorhanden sind. Sie hängen zusammen mit folgenden Aspekten der Internet-Kommunikation:

- Im Internet werden Daten in bislang nicht gekannter Schnelligkeit zu Adressaten an allen Orten der Welt übermittelt. Die Tastatur dient als Schreibwerkzeug, der Bildschirm als Lesefläche. Das erhöhte schriftliche Kommunikationsaufkommen wird kompensiert durch die Beschleunigung des Lesens und Schreibens. Einige der Auswirkungen davon sind in 4.1. behandelt.

- Speziell in den Kommunikationsdiensten des Internet hat sich ein medientypischer kommunikativer Stil (i.S. von Kallmeyer, 1995) herausgebildet, dessen Leitlinien in der sog. „Netikette“, eine Zusammenziehung von „Netz" und „Etikette“, festgehalten und tradiert werden. Teil dieses kommunikativ-sozialen Stils sind gruppensprachliche Elemente, die in 4.2. vorgestellt.

\subsection{Schnelligkeit und Flüchtigkeit}

Ob sich durch die Übertragungsschnelligkeit des Internet wirklich Arbeitsabläufe effektiver und zeitsparender gestalten lassen, ob das Internet nicht eher ein Zeitverschwendungs-Medium ist, wie z.B. Stoll (1996) behauptet, hängt ab von der Medienkompetenz der Individuen und Institutionen, die das Netz nutzen. Tatsache ist, daß sich durch die Netzkommunikation für viele das Aufkommen schriftlich fixierter Kommunikation deutlich erhöht: Wenn Email nicht nur als schnelle Alternative zur gelben Post, sondern auch als 
Alternative zum Telefon genutzt wird (z.B. für kurze Anfragen, Terminabsprachen etc.), verlagert sich ein Teil der bislang mündlich geführten Gespräche ins schriftliche Medium. Durch die neuen Kommunikationsformen ergeben sich aber auch beruflich und privat neue Schreibanlässe: Die Fachdiskussion in Mailinglisten und Foren, die Beteiligung am Chat, der Entwurf einer eigenen privaten Homepage etc.

Das erhöhte Aufkommen an schriftlicher Textproduktion wird vielfach dadurch kompensiert, daß weniger Zeit in die sprachlichen Formulierungen und deren Kontrolle investiert wird. Diese Beschleunigung des Schreibens hinterläßt deutliche Spuren in den Produkten (vgl. Jakobs, 1998). Auffällig sind zunächst viele Verdreher und Tippfehler, die für die schnelle Eingabe mit der Tastatur typisch sind. Auch viele Orthographie- und Satzbaufehler (z.B. „das“ statt „daß“ im zweiten Turn in Abbildung 3) dürften nicht auf mangelhafte Rechtschreib- und Grammatikkompetenz zurückzuführen sein, sondern sind, wie der Vergleich anderer Texte desselben Produzenten oft zeigt, eher Performanzfehler bei der raschen und spontanen Herstellung von schriftlichen Äußerungen, die ohne genaue Korrektur und Überprüfung direkt nach der Fertigstellung abgeschickt werden. An der Anzahl der Fehler läßt sich am besten ablesen, wieviel Zeit sich der Schreiber genommen hat, um seine Formulierungsprodukte vor dem Absenden zu überprüfen. Die Bandbreite reicht von gut überprüften privaten und institutionellen Emails (vgl. 2.1.) über flüchtige Emailnotizen und Forenbeiträge hin zu den Turns im Chat, bei dem es letztlich darum geht, die Tippgeschwindigkeit der Sprechgeschwindigkeit anzunähern oder diese durch Abkürzungen (s.u.) gar zu übertrumpfen.

Bei den Rezipienten besteht im Normalfall die Disposition, über solche Fehler hinwegzulesen. Einerseits ist die Rezeption am Bildschirm ebenfalls flüchtiger, andererseits ist es Teil des kommunikativ-sozialen Stils im Internet, Fehler zu tolerieren, zumindest solange dadurch die Verständlichkeit nicht gefährdet ist (vgl. Pansegau, 1997). Netzkommunikation wird verstanden als Möglichkeit, sich schnell, unkompliziert und auf informelle Weise miteinander auszutauschen. Die Lebenszeit der „Netzbürger“ gilt als wertvolle und schützenswerte Ressource, auf die in den Regeln der Netikette Bezug genommen wird ${ }^{7}$. Die Grundregel für die Verfertigung von Forenbeiträgen lautet ,kurz und prägnant" (,short and to the point") und muß vor dem Hintergrund als eine medienspezifische Facette der ,Zeit-in-Geld-Umrechnung" verstanden werden, die für die Konzeptmetapher „Zeit-ist-Geld“ (vgl. Liebert, 1992, S. 137) generell typisch ist: Wer vom heimischen Rechner aus auf das Netz zugreift, muß für die Zeit, in der Daten übertragen werden, bezahlen. Die Kosten für die Zeit, die das Herunterladen irrelevanter oder in-

7 So schreibt beispielsweise Shea (1996): ,When you send email or post to a discussion group, you're taking up other people's time (or hoping so). It's your responsibility to ensure that the time they spend isn't wasted“" (Rule 4). 
formationsloser Beiträge oder Dateien im WWW dauert, sind also in sehr konkreter Weise in Geldwert zu messen. Inhaltliche Relevanz, Aktualität und Originalität werden deshalb höher bewertet als Konformität mit den Normen bildungsbürgerlicher Schriftkultur. Es gilt mithin als oberlehrerhaft und unpassend, Orthographie- und Grammatikfehler anderer zu kritisieren (vgl. Shea, 1996). Die Frage „was heißt destikulierend“ im Chat-Beispiel in Abbildung 4 ist deshalb wohl als Neckerei zu verstehen, die genau auf dem Konsens aufsetzt, daß Tippfehler beim schnellen Chatten fast unvermeidbar sind.

\subsection{Gruppensprachliche Wortschatz- und Zeichenkonventionen}

Wenn von der „Sprache des Internet“ die Rede ist, sind oft Sprachelemente, Abkürzungen und ASCII-Zeichensysteme gemeint, durch deren Kenntnis und Verwendung sich eingeweihte Netzbürger (,netizens") vom uneingeweihten Einsteiger (,newbies“) sprachlich absetzen können. Viele dieser Elemente stammen aus der Gruppensprache der sog. „Hacker“, die schon seit langem Computernetzwerke zur Kommunikation nutzen ${ }^{8}$. Wesentliche Elemente dieser Gruppensprache sind Abkürzungen und Akronyme. $\mathrm{Zu}$ den häufig verwendeten gehören: IMO (,,in my opinion“) bzw. IMHO (,,in my humble opinion“), LOL (,laughing out loud“) bzw. ROTFL (,rolling on the floor laughing"), BTW (,by the way"). In der Gründerzeit waren solche Kurzformen sinnvoll, um die Übertragungszeit (und damit Übertragungskosten, s.o.) zu sparen. Durch die Erhöhung der Übertragungskapazitäten spielt dieser Aspekt inzwischen bei Textdateien eine untergeordnete Rolle. Abkürzungen dienen nun vornehmlich dem Zweck, Tippzeit für häufig genutzte Floskeln zu sparen und die Identität der Netzgemeinde zu stärken, d.h. Außenseiter und Einsteiger als solche erkennbar zu machen. Viele der Abkürzungen und Akronyme stammen aus dem Englischen, werden aber auch im deutschsprachigen Internet genutzt: Hierzu gehört die Abkürzung „RTFM“ - Read that fucking manual - als Antwort auf das Hilfegesuch des Teilnehmers „"'amour": ,hey ihr bin neu hier, need help!“ in Abbildung 4. Da ein Newbie RTFM vermutlich nicht richtig auflösen kann, muß allerdings bezweifelt werden, ob die Zurechtweisung ernst gemeint ist, ob es sich nicht eher um einen Versuch handelt, einen „Newbie-Simulanten“ zu entlarven, der durch vorgetäuschte Hilfsbedürftigkeit versucht, seinem im Namen schon angedeuteten Kommunikationsziel näher zu kommen.

Aus der Notwendigkeit, Übertragungskapazitäten zu schonen, entwikkelten sich bald neue Kürzungsstrategien, die zeigen, daß der kommunikative Stil im Internet von Menschen geprägt ist, die zwar den bildungsbürgerlichen

8 Vgl. das „New Hacker’s Dictionary“ unter http://sagan.earthspace.net/jargon/jargon_toc. html. 
Schriftnormen wenig Respekt entgegenbringen, denen es aber nicht an sprachlicher Kreativität mangelt. Eine Strategie spielt mit dem Verhältnis von medialer Mündlichkeit und Schriftlichkeit, indem sie die lautliche Realisierung von Zahlen oder Buchstaben schriftlich fixiert. Beispiele sind CU (,see you“), 4 you ("for you") oder Hab 8. Die Freude am spielerischen Umgang mit Sprache zeigt sich auch im sog. ,Soundalike Slang“, bei dem Wörter oder Akronyme parodistisch abgewandelt werden. So wird z.B. das unter Computerfreaks und Systemverwaltern unbeliebte Betriebssystem „Windows“ zu „Windoof", die „Telekom“ trotz guter Börsenwerte zu „Telekotz“. Systemverwalter und Hotline-Bedienstete bezeichnen ausgewählte Exemplare ihrer Kundschaft - in Anlehnung an den bekannten nuklearen GAU - als „DAU“ (,dümmster anzunehmender User“, vgl. Haase, Huber, Krumeich \& Rehm, 1997, S. 72).

Viel diskutiert werden in der Literatur zur Netzsprache die "Smileys“ genannten Emotikons. Es handelt sich um ,ikonographische Rekonstruktionen typisierter Gesichtsausdrücke" (Beißwenger, 1999, S. 86) die ausschließlich mit ASCII-Zeichen produziert werden. Der jeweilige Gesichtsausdruck wird erkennbar, wenn man den Kopf nach links dreht. Das Emotikon ,,--)“ drückt beispielsweise Zufriedenheit, Lächeln und freundliche Gesinnung aus; andere Emotikons stehen für Enttäuschung oder Ärger; für Langeweile oder Gleichgültigkeit und für Übermüdung durch zu lange Bildschirmarbeit. Eine wichtige Funktion hat dabei der sog. „,winking smiley“, ,;-)“. Er fungiert als Indikator für uneigentliches Sprechen und soll nach dem Wunsch der Netikette einem ironisch gemeinten Statement beigegeben werden, um Mißverständnisse und daraus resultierende Flame-Wars (vgl. 3.2) zu vermeiden.

Emotikons gelten als Mittel, um das Fehlen von Mimik und Gestik in konzeptionell mündlichen Kommunikationsformen des Internet zu kompensieren (vgl. Lenke \& Schmitz, 1995; Haase, Huber, Krumeich \& Rehm, 1997). Allerdings gibt es grundlegende Unterschiede zwischen Emotikons und natürlicher Mimik und Gestik: Natürliche Mimik und Gestik sind nur in begrenztem Ausmaß willentlich kontrollierbar; Emotikons hingegen werden gesetzt ${ }^{9}$. Während Mimik und Gestik im Face-to-Face-Gespräch nicht ausgeblendet werden können, ist der Einsatz von Emotikons optional. Es handelt sich also um intentional gesetzte Zeichen, die in vielfältigen Formen und Funktionen ${ }^{10}$ zur Beschreibung von Gefühlen und Aussehen eingesetzt werden können.

9 Für diesen Hinweis danke ich Hans-Jürgen Bucher.

10 Zur Klassifikation von Emotikons vgl. Beißwenger (1999) und Runkehl, Schlobinski \& Siever (1998); eine Aufstellung des Inventars findet sich z.B. unter http://www. eff.org/papers/eegtti/eeg_286.html. 


\section{Fazit}

Kehren wir zurück zu der eingangs gestellten Frage: Sind die Erscheinungsformen konzeptioneller Mündlichkeit in der Internet-Kommunikation eine qualitativ neue Entwicklung oder handelt es sich lediglich um eine weitere Facette eines generellen Trends, der z.B. in Sieber (1998) als „Veränderung kommunikativer Grundmuster der Schriftlichkeit" beschrieben wurde. Auf der Basis der bisherigen Ausführungen möchte ich dazu die folgende Position zur Diskussion stellen:

Wenn bislang von einer Vermündlichung von Schriftsprache die Rede war $^{11}$, ging es meist darum, daß sich Texte derselben schriftlichen Textsorte über eine Zeitspanne hinweg verändert haben, daß sie zunehmend Merkmale aufweisen, die im zweiten Abschnitt als typisch für konzeptionelle Mündlichkeit genannt worden sind. Die Beobachtung war also, daß Texte bei gleichbleibendem kommunikativem Setting anders gestaltet werden. Sieber (1998) zeigt diese Veränderungen sehr eindrücklich an einem Corpus von Aufsatztexten, die zwischen 1881 und 1991 entstanden sind. In der schriftlichen Kommunikation im Internet hingegen entwickeln sich, das sollten die vorigen Abschnitte deutlich gemacht haben, neue Kommunikationsformen heraus, die von ihren technischen Randbedingungen her - Übertragungsschnelligkeit, Softwarefunktionen wie „Zitieren“, technisiertes Turntaking beim Chat Dialogizität in medialer Schriftlichkeit in qualitativ neuer Weise fördern, die deshalb von vornherein stärker am Pol der Nähe (zeitlicher Nähe, metaphorisch konstituierte Nähe) in dem von Koch und Oesterreicher (1994) skizzierten Nähe-Distanz-Kontinuum anzusiedeln sind. Die Merkmale konzeptioneller Mündlichkeit können dann zunächst einfach als sprachlicher Reflex dieser technikinduzierten Verschiebung hin zum Nähepol interpretiert werden; sie erlauben aber noch keine Rückschlüsse auf eine veränderte kommunikative Grundhaltung der Menschen, die die neuen Kommunikationsformen nutzen.

Die Einordnung in das Nähe-Distanz-Kontinuum muß zwischen den verschiedenen Kommunikationsformen Email, Foren, Chat differenzieren. Vom kommunikativen Setting her steht sicherlich der simultane Chat dem Nähepol am nächsten. Trotz der in 3.3. erwähnten Unterschiede ähnelt der Chat mehr dem Face-to-Face-Gespräch oder dem Telefonat als den situationsentbundenen Textsorten, die man gewöhnlich mit Schriftlichkeit in Verbindung bringt. Und gerade beim Chat zeigt sich ein mediengeschichtlich interessantes Phänomen am deutlichsten: Die Entwicklung der Schriftlichkeit läßt sich als eine Geschichte des sprachlichen Ausbaus beschreiben, ein Ausbau, der darauf abzielt, den Anforderungen gerecht zu werden, die mit der Loslösung sprachlicher Äußerungen von der Äußerungssituation einher gehen (vgl. Koch \&

11 Ich beziehe mich auf die Überblicksdarstellungen in Sieber (1998) und Polenz (1999). 
Oesterreicher, 1994). Die in 4.2. erläuterten sprachlichen und semiotischen Innovationen setzen diesen Ausbau unter neuen Vorzeichen fort: Nun geht es darum, mit getippt-schriftlichen Mitteln Elemente der Face-to-Face-Kommunikation - Mimik, Gestik, Handlungsbeschreibungen - zu integrieren, die für die technisierte Form schriftlicher Simultankommunikation benötigt werden.

Das Neue an der Foren-Kommunikation ist nicht so sehr die zeitliche Nähe - hier können u.U. mehrere Tage vergehen, bis auf einen Beitrag reagiert wird - sondern die soziale Nähe, die dem Selbstverständnis vieler Forenteilnehmer entspringt, eine „virtuelle Gemeinschaft" mit eigenen, in der Netikette kodifizierten Verhaltensregeln und Wertsetzungen und einer gemeinsamen Kommunikationsgeschichte zu sein. Die in der Netikette tradierten Grundwerte der „Netzgemeinde“ - Meinungsfreiheit, kostenloser Austausch von Ressourcen und Wissen, Toleranz - sind zwar nicht identisch, aber doch bestens kompatibel mit den Grundhaltungen, die Sieber (1998) für den Wandel von Mustern in schriftlichen Schülertexten verantwortlich macht: Hohe Ansprüche an Direktheit und Authentizität, das Bemühen, Distanz abzubauen und sich als individuelle Person mit eigenen Erfahrungen einzubringen und - damit einhergehend - mit einer abnehmenden Disposition, Vorbildern elaborierter Schriftlichkeit nachzueifern und deren Wissen zu reproduzieren. Der kommunikativ-soziale Stil der Foren-Kommunikation kommt dieser Grundhaltung entgegen, indem inhaltliche Relevanz, Aktualität und Engagement für das Anliegen des Forums höher bewertet werden als sprachliche und formale Korrektheit und Elaboriertheit.

Hier genau liegt nun die Schnittstelle, über die sich m.E. die konzeptionelle Mündlichkeit in der Netzkommunikation an den allgemeinen Wandel im Umgang mit Schriftlichkeit anschließen läßt: Ganz im Sinne der von Holly (1996) vertretenen ,mediendarwinistischen“ These, daß sich neue Medien vor allem dann durchsetzen, wenn sie bestimmten Kommunikationsbedürfnissen besser entgegen kommen als bislang vorhandene Medien dies tun, kann man den Erfolg der Forenkommunikation darauf zurückführen, daß deren kommunikativ-sozialer Stil der von Sieber (1998) beschriebenen kommunikativen Grundhaltung der Mündlichkeit bestens entspricht. Weitere Erfolgsfaktoren sind sicherlich die wachsende Bedeutung des raschen Informationsaustausches unter Experten sowie zwischen Experten und Laien in einer Zeit schnell anwachsenden und sich verändernden Wissens, aber auch die Mischung zwischen Zugehörigkeit und Unverbindlichkeit, die für „virtuelle Gemeinschaften" typisch ist.

Auch der Erfolg der Email-Kommunikation läßt sich aus der Sicht der o.g. kompensatorischen Medienentwicklungsthese erklären: In einer Gesellschaft, in der viele Menschen Lebenszeit als knappe Ressource empfinden, erscheint ein Medium schnell unentbehrlich, das es erlaubt (vgl. Abbildung 1), einen kommunikativen Vorgang mit einem Satz abzuschließen, für den bislang eine ausführlichere Bezugnahme nötig gewesen wäre - und sei es nur, weil ein einzelner Satz auf einem Blatt Papier so verloren aussieht - oder 
ein Telefonat hätte geführt werden müssen, bei dem das eigentliche Anliegen (bei Einhaltung gewisser Höflichkeitsstandards) auch erst nach einer sozialen Warmlaufphase angesprochen werden kann.

$\mathrm{Ob}$ und wie der neue Umgang mit Schriftlichkeit in den verschiedenen Internet-Diensten auf Dauer die Schriftlichkeit außerhalb des Netzes beeinflußt, kann momentan noch nicht beurteilt werden; für eine Vergleichsstudie ist die Netzkommunikation noch zu jung und unterliegt, durch das rasche Wachstum des Internet, zu starken Veränderungen. Bislang gibt es eigentlich keinen Grund zur Annahme, daß der pragmatische Umgang mit Schriftlichkeit, der vor allem auf schnelle und effiziente Kommunikation setzt und dabei auf Elaboration verzichtet, negative Auswirkungen auf die Anfertigung von Schrifttexten hat, bei denen Textplanung und sprachliche Elaboration wichtig sind. Zumindest bei einigen Teilnehmern der „sci.“-Foren (,sci“" steht für „science“) läßt sich nachweisen, daß sie durchaus in der Lage sind, verständliche und sprachlich sehr gut gemachte wissenschaftliche Artikel zu schreiben, auch wenn die Forenbeiträge alle in Abschnitt genannten Merkmale aufweisen.

Vieles spricht dafür, die neuen Kommunikationsformen positiv als Erweiterung der medialen Optionen zu werten, die für die Erreichung kommunikativer Handlungsziele zur Verfügung stehen. Aus dieser Perspektive wird es für das erfolgreiche Kommunizieren zunehmend wichtig werden, die passende Kommunikationsform für einen gegeben Zweck zu finden und die darin üblichen Gepflogenheiten zu kennen. Es gilt also eine ,muttersprachliche Mehrsprachigkeit “"12 zu entwickeln und dabei die verschiedenen Erscheinungsformen von Schriftlichkeit zu berücksichtigen. Hierfür auf wissenschaftlicher Basis Prinzipien und Strategien zu entwickeln und diese im Bildungsbereich vermittelbar zu machen, ist eine Herausforderungen für die Schriftlichkeitsforschung sowie die Kommunikations- und Medienwissenschaft der kommenden Jahre.

\section{Literatur}

Beißwenger, M. (1999). Kommunikation in virtuellen Welten: Sprache, Text und Wirklichkeit. Heidelberg: Magisterarbeit, Neuphilologische Fakultät der Universität Heidelberg. Internet: http://www.gs.uni-heidelberg.de/ beisswen/webchat/.

Ehlich, K. (1994). Funktion und Struktur schriftlicher Kommunikation. In: H. Günther \& O. Ludwig (Hg.), Schrift und Schriftlichkeit. Ein interdisziplinäres Handbuch internationaler Forschung (1. Halbband, S. 18-41). Berlin: de Gruyter.

12 Unter „muttersprachlicher Mehrsprachigkeit“ versteht Sieber (1998, S. 219) „die Fähigkeit in verschiedenen Situationen und in Bezug auf unterschiedliche Adressaten aus den verschiedenen Registern, Fach- und Gruppensprachen eine sinnvolle und angemessene Wahl zu treffen“. 
Feldweg, H. Kibiger, R. \& Thielen, C. (1995). Zum Sprachgebrauch in deutschen Newsgruppen. Osnabrücker Beiträge zur Sprachtheorie, 50, 143-154.

Günther, U. \& Wyss, E.L. (1996). Email-Briefe - eine neue Textsorte zwischen Mündlichkeit und Schriftlichkeit. In E.W. Hess-Lüttich, W. Holly, \& U. Püschel (Hg.), Textstrukturen im Medienwandel (S. 61-86). Frankfurt am Main: Peter Lang.

GVU-10 (1988). GVU's Tenth WWW User Survey (Conducted October 1998). Internet: http://www.gvu.gatech.edu/user_surveys/survey-1998-10/.

Haase, M., Huber, M., Krumeich, A. \& Rehm, G. (1997). Internetkommunikation und Sprachwandel. In R. Weingarten (Hg.), Sprachwandel durch Computer (S. 51-85). Opladen: Westdeutscher Verlag.

Hess-Lüttich, E. W. (1997). E-Epistolographie: Briefkultur im Medienwandel. In A. Hepp \& R. Winter (Hg.), Kultur-Medien - Macht: Cultural Studies und Medienanalyse (S. 225-246). Opladen: Westdeutscher Verlag.

Holly, W. (1992). Secondary orality in the electronic media. In U. Quasthoff (Ed.), Aspects of oral communication (pp. 340-363). Berlin: de Gruyter.

Holly, W. (1996). Zur inneren Logik der Mediengeschichte. In B, Rüschoff \& U. Schmitz (Hg.), Kommunikation und Lernen mit alten und neuen Medien (S. 9-16). Frankfurt am Main: Peter Lang.

Hoffmann, G, \& Kuhlmann, D. (o.J.). Regulierung und Selbstregulierung im Internet. Netiquette, Nutzungsregeln, Rechtsvorschriften, Verträge (http://prz.tuberlin.de/ derek/internet)

Hoffmann, U. (1997). Panic Usenet. Netzkommunikation in (Un)ordnung (http://duplox. wz-berlin.de/texte/panic).

Jakobs, E.-M. (1998). Mediale Wechsel und Sprache. Entwicklungsstadien elektronischer Schreibwerkzeuge und ihr Einfluß auf Kommunikationsformen. In W. Holly \& B.U. Biere (Hg.), Medien im Wandel (S. 187-209). Opladen: Westdeutscher Verlag.

Kallmeyer, W. (1995). Zur Darstellung von kommunikativem sozialem Stil in soziolinguisitischen Gruppenporträts. In I. Keim (Hg.), Kommunikation in der Stadt. Teil 3: Kommunikative Stilistik einer sozialen Welt „kleiner Leute“ in der Mannheimer Innenstadt (S. 1-25). Berlin: de Gruyter.

Koch, P. \& Oesterreicher, W. (1994). Schriftlichkeit und Sprache. In H. Günther \& O. Ludwig (Hg.), Schrift und Schriftlichkeit. Ein interdisziplinäre Handbuch internationaler Forschung (1.Halbband, S. 587-604). Berlin: de Gruyter.

Kuhlen, R. (1995). Informationsmarkt. Chancen und Risiken der Kommerzialisierung von Wissen (= Schriften zur Informationswissenschaft 15). Konstanz: UVK.

Lenke, N. \& Schmitz, P. (1995). Geschwätz im ,Globalen Dorf‘ - Kommunikation im Internet. Osnabrücker Beiträge zur Sprachtheorie, 50, 117-141.

Liebert, W.-A. (1992). Metaphernbereiche der deutschen Alltagssprache. Kognitive Linguistik und die Perspektiven einer kognitiven Lexikographie. Frankfurt am Main: Peter Lang.

Pansegau, P. (1997). Dialogizität und Degrammatikalisierung in Emails. In R. Weingarten (Hg.), Sprachwandel durch Computer (S. 86-104). Opladen: Westdeutscher Verlag.

Partl, H. (o.J.). „Nettigkeit“ für die Menschen im UseNet (http://www.kirchwitz.de/amk/dni/erst-lesen-dann-schreiben).

Polenz, P.v. (1999). Deutsche Sprachgeschichte vom Spätmittelalter bis zur Gegenwart. Band III: 19. und 20. Jahrhundert. Berlin: de Gruyter.

Quasthoff, U.M. (1997). Kommunikative Normen im Entstehen: Beobachtungen zu Kontextualisierungsprozessen in elektronischer Kommunikation. In R. Weingarten ( $\mathrm{Hg}$.), Sprachwandel durch Computer (S. 23-50). Opladen: Westdeutscher Verlag.

Runkehl, J., Schlobinski, P. \& Sieber, T. (1998). Sprache und Kommunikation im Internet. Überblick und Analysen. Opladen: Westdeutscher Verlag. 
Shea, V. (1996). The core rules of netiquette. (http://www.albion.com/netiquette/corerules.html)

Sieber, P. (1998). Parlando in Texten. Zur Veränderung kommunikativer Grundmuster in der Schriftlichkeit (= Reihe Germanistische Linguistik 191). Tübingen: Niemeyer.

Stoll, C. (1996). Die Wüste Internet. Geisterfahrten auf der Datenautobahn. Frankfurt am Main: Fischer.

Storrer, A., Feldweg, H. \& Hinrichs, E.H. (1993). Korpusunterstützte Entwicklung lexikalischer Wissensbasen. Sprache und Datenverarbeitung, 17, 59-72.

Storrer, A. \& Waldenberger, S. (1998). Zwischen Grice und Knigge: Die Netiketten im Internet. In H. Strohner, L. Sichelschmidt \& M. Hielscher (Hg.), Medium Sprache (= Forum Angewandte Linguistik 34, S. 63-77). Frankfurt am Main: Peter Lang.

Wyss, E.L. (1999). Iconicity in the digital world. An opportunity to create a personal image? In M. Nänny \& O. Fischer (Hg.), Form miming meaning. Iconicity in language and literature (S. 285-304). Amsterdam: John Benjamins Publishing Company.

Zifonun, G. \& Hoffmann, L., \& Strecker, B. (Red.) (1997). Grammatik der deutschen Sprache. Berlin: de Gruyter. 